\title{
Dermatoses of Pregnancy
}

\author{
Yuval Ramot • Arieh Ingber \\ Published online: 7 September 2012 \\ (C) Springer Science+Business Media, LLC 2012
}

\begin{abstract}
Pregnancy is frequently associated with major skin changes. Some of these changes are attributed to pregnancyspecific dermatoses, which often are overlooked and misdiagnosed by physicians. We discuss four major pregnancyspecific dermatoses: pemphigoid gestationis; pruritic urticarial papules and plaques of pregnancy; prurigo of pregnancy; and pruritic folliculitis of pregnancy. Accurate diagnosis and management is crucial, not only to alleviate symptoms, but also to estimate correctly the risk for the fetus.
\end{abstract}

Keywords Pregnancy $\cdot$ Pemphigoid gestationis $\cdot$ Pruritic urticarial papules and plaques of pregnancy $\cdot$ Prurigo of pregnancy $\cdot$ Pruritic folliculitis of pregnancy

\section{Introduction}

Pregnancy is a period in a woman's life that is full with changes. Many of these changes are related to the skin, and some of these changes may have great impact on the woman's life. Changes in the skin during pregnancy may be divided into physiological changes and pregnancy-specific dermatoses. While many physicians are familiar with the normal physiologic changes in pregnancy, knowledge on the rarer pregnancy-specific dermatoses is oftentimes lacking. This may lead to misdiagnosis and mistreatment of the patients. The purpose of the current review is to focus on some of the more important and common pregnancyspecific dermatoses: pemphigoid gestationis (PG); pruritic urticarial papules and plaques of pregnancy (PUPPP); prurigo of pregnancy (PP); and pruritic folliculitis of pregnancy (PFP) - highlighting the differences between these conditions. We hope that this will help to better diagnose, and hence to better treat, pregnant patients with these disorders.

\section{Y. Ramot $\cdot$ A. Ingber $(\bowtie)$}

Department of Dermatology,

Hadassah - Hebrew University Medical Center,

PO Box 12000, Jerusalem 91120, Israel

e-mail: arieh@hadassah.org.il

\section{Pemphigoid Gestationis}

PG is a rare, autoimmune, blistering disease of pregnancy [1]. Its incidence varies between 1 in 2,000 to 1 in 60,000 pregnancies, depending on the presence of HLA-haplotypes $\mathrm{B} 8, \mathrm{DR} 3$, and DR4 in the mother and DR2 in the father [2-6]. Other autoimmune diseases, including Grave's disease (most common), Hashimoto thyroiditis, vitiligo, autoimmune thrombocytopenia, and pernicious anemia, have been reported in association with PG $[4,7]$.

PG is initiated by the identification of placental proteins as foreign antigens and subsequent production of antiplacental antibodies of the subclass IgG1 [1,4]. These antibodies cross-react with similar proteins of the skin basement membrane [1]. The main antigen has been recognized to be collagen XVII (also termed BP180 or BPAg2), which is found in both skin and placenta, and especially important in this condition is the NC16A domain, similar to bullous pemphigoid $[4,8]$. Less frequently, BP230 is the target antigen [9]. The antigens are presented to the maternal immune system by an abnormal expression of major histocompatibility complex class II molecules [8]. This initiates a cascade of reactions involving TH2 lymphocytes, cytokines, and polymorphonuclear cells, leading to blister formation [8]. Recently, both IgA and $\operatorname{IgE}$ antibodies have been reported in PG [10, 11].

Symptoms usually begin between 21 and 28 weeks of gestation and 1 week postpartum, and recover a few weeks following delivery [8]. It can very rarely appear in the context of trophoblastic tumors (choriocarcinoma, hydatidiform mole) [4]. Onset of symptoms in early pregnancy is usually associated with a better prognosis and termination of rash earlier after delivery [12••, 13].

Characteristic presentation is of an abrupt intensely pruritic, periumbilical skin eruption, which consists of erythematous urticarial papules and plaques [2-4]. The rash quickly spreads, but spares the face, scalp, and mucous membranes $[2,3,8]$. In that phase of the disease, it is very difficult to differentiate PG from PUPPP. When blisters start to form, diagnosis of $P G$ is much easier, although cases without blistering also have been described [4, 6]. Blisters often 
contain clear or sometimes blood-stained fluid [8]. The bullae rupture, leaving erosions that are covered with yellowish or hemorrhagic crusts, and culminating in residual pigmentation and rarely cicatrization [5]. Nikolsky sign may be positive [5]. Systemic symptoms have been described and include lassitude, exhaustion, fever, chills, and psychological stress associated with the unbearable pruritus [5]. Exacerbations and remissions are characteristically evident during the course of pregnancy, with marked improvement in late pregnancy [4]. Nevertheless, in $75 \%$ of cases there is an exacerbation at delivery [4]. Resolution time after delivery is usually 4 weeks for the bullous eruption and 14 weeks for the urticarial rash [5].

Relapses are reported in subsequent pregnancies, during the first menstrual cycles, or with the use of oral contraceptives [1]. In subsequent pregnancies, the condition can present earlier and be more aggressive. Disease-free pregnancies also have been reported but only very rarely $(5-8 \%)[2,4,7,14]$. Changing partners has not been associated with an increased risk of developing PG, but it is unknown whether it changes the risk of recurrence [14]. PG is associated with a high stillbirth rate, premature labor, and delivery of low-birthweight infants $[15,16]$. Abortions have been reported in approximately $20 \%$ of cases [17]. This risk correlates with disease severity and not with corticosteroid treatment [18]. The rash may spread to the fetus in $5-10 \%$ of cases, probably due to passive antibody transfer in utero, and present as papular and bullous lesions [2, 3, 7]. The rash in these infants is usually mild and self-resolving within days to weeks and usually does not evolve after birth $[2,4,7]$.

Histopathology findings depend on the stage and severity of the disease [4]. Early lesions reveal epidermal and papillary dermal edema, together with perivascular inflammatory infiltrate rich with lymphocytes, histiocytes, and eosinophils [4]. Later, basal cell necrosis at the tip of the dermal papilla may be evident $[7,17]$. In the bullous stage, subepidermal blistering containing numerous eosinophils can be found, located to the lamina lucida of the dermal-epidermal junction (DEJ) $[2-4,7]$.

By immunofluorescent studies of perilesional skin, linear complement $\mathrm{C} 3$ deposition can be found along the DEJ in $100 \%$ of cases [4]. IgG can be demonstrated in $25-30 \%$ of women affected $[2-4,7,8]$. The same features can be found in skin samples from affected babies and also in the placenta. The anti-BP180 titer is usually high in the beginning of the disorder and decreases in remission. Therefore, frequent testing facilitates diagnosis and follow-up [19, 20].

Laboratory tests are usually normal, although eosinophilia may be seen in as many as $50 \%$ of patients and can correlate with disease severity [5]. Other parameters that can be elevated include immunoglobulin levels, erythrocyte sedimentation rate, acute phase proteins levels, and antithyroid antibodies [5].

Treatment is determined according to the severity of the disease, and its goal is to control pruritus and to prevent the formation of blisters [4]. It is advised to eliminate contact with affected sites to minimize the risk of secondary infection and scarring, and large blisters may be drained [15, 21]. Systemic corticosteroids are the treatment of choice, and doses of $0.5-1 \mathrm{mg} / \mathrm{kg} /$ day of prednisolone are recommended $[1,4]$. Mild cases can be treated with ultrapotent topical corticosteroids, emollients, and antihistamines. Immunoapheresis can be successfully used in cases of PG that are resistant to conventional treatment regimens [22]. Other treatment options for the postpartum period in resistant cases may include tetracyclines, nicotinamide, cyclophosphamide, cyclosporine, pyridoxine, gold, methotrexate, azathioprine, dapsone, rituximab, intravenous immunoglobulins, or chemical oophorectomy with goserelin [7-9, 22-27].

\section{Pruritic Urticarial Papules and Plaques of Pregnancy}

PUPPP is the most common pregnancy-specific dermatosis [28] and has been reported to occur in 1 of 130-300 pregnancies [28]. This is a benign, self-resolving, pruritic, inflammatory disorder. In addition to PUPPP (the term most commonly used in the United States), this condition has been termed polymorphic eruption of pregnancy (the term preferred in Great Britain) [29], toxemic rash of pregnancy [30, 31], late onset prurigo of pregnancy [31], Bourne's toxemic rash of pregnancy, and toxic erythema of pregnancy [32].

The rash of PUPPP usually first appears on the abdomen, often along the striae, and later spreads to the buttocks and proximal thighs $[4,33]$. In severe cases, it can quickly become generalized, but systemic symptoms are almost always absent $[4,12 \bullet \cdot$. The rash starts with 1- to 2-mm urticarial papules, surrounded by a narrow and pale hallo, that coalesce into plaques [34]. It is characterized by intense pruritus, which can be very bothersome to the patients, and even interrupt their sleep and lives [33, 35]. A helpful clue to differentiate this condition from PG is the characteristic sparing of the umbilical region [4]. Sparing of the face and acral sites also is characteristic, although one case of extensive palmoplantar involvement has been reported [36, 37•]. Mucous membranes are usually not involved [38]. In half of the patients, the rash can develop into vesicles (and rarely even bullae), widespread erythema (nonurticated), and targetoid and eczematous rash [4, 37•, 39]. It develops during the third trimester in 85-91\% of patients (mean onset at 35 weeks of gestation), although in up to $15 \%$ of patients it can start immediately postpartum, and onset as late as 4 weeks postpartum has been described $[4,33,34,37 \bullet, 40]$.

The disorder is more common with first pregnancies (73\%), excessive maternal weight gain, and multiple gestations $[4,37 \bullet, 41]$. Additionally, familial occurrences have been reported [41].

Although it is a common disorder, the etiology of PUPPP is still a mystery. It has been suggested that there is a connection 
between this disease and the maternal immune system and fetal cells [42]. Additionally, the increased incidence in women with multiple gestations and increased maternal weight gain, together with the fact that the rash starts on the striae distensae, suggest that skin stretching may have a contributing role in initiating an immune-mediated reaction [4, 17, 43]. In accordance with this theory, there is an increase in CD1a cells in the inflammatory infiltrate, therefore suggesting that structures that were previously inert develop antigenic properties [4]. Previously suggested theories, such as immunological changes, placental products, increased birth weight, or male sex of the newborn, have not been confirmed [34, 37•, 44•, 45, 46]. There is no HLA-antigen association in women with PUPPP [47]. The association of PUPPP with multiple gestations has led to the assumption that there is a role for sex hormones in the pathogenesis of this disorder (i.e., increased estrogen and progesterone levels) [48], and indeed, progesterone has been found to exacerbate the inflammatory process in the tissue level, and increased progesterone receptor immunoreactivity has been evident in skin lesions of PUPPP [47, 49]. However, it should be stressed that in most studies levels of sex hormones in PUPPP patients were not different from controls [34, 45, 50].

PUPPP is a clinical diagnosis, and a skin biopsy is seldom needed. Histopathologic findings are generally nonspecific and change with the stage of disease [4, 41]. Early lesions show superficial perivascular lymphohistiocytic infiltrate together with eosinophils and a prominent dermal edema [4]. Older lesions show epidermal changes, which include spongiosis and hyper- and parakeratosis [4]. In case of vesicles or bullae, immunofluorescence studies might be warranted, both direct and indirect, and these studies are usually negative [4]. Vascular deposits of IgM, IgA, and $\mathrm{C} 3$ have been observed in some patients [51, 52].

Differential diagnosis might include contact dermatitis, a viral exanthem, toxic drug eruption, herpes gestationis, PP, PFP, insect bites, scabies infestation, urticarial allergic reaction, pityriasis rosea, and rarely erythema multiforme [32-34, 37•, 47, 53-55].

There is no specific treatment, and management is usually symptomatic. Small doses of antihistamines, bland topical emollients, and high-potency topical steroids can be used to treat symptoms. Systemic steroids are usually very effective for severe pruritus, and customary treatment is with prednisone in doses of 40-60 $\mathrm{mg} / \mathrm{d}$ with tapering [4, $41,53,55,56]$. Reassurance of the mother is an important part of management. Delivery usually does not lead to cessation of intractable symptoms [47].

PUPPP is not associated with adverse maternal or fetal outcomes, and there is no cutaneous involvement of the newborn [41]. It should be noted, however, that one study described increased maternal weight gain and increased newborn birth weight [43]. The rash characteristically disappears 1 to 2 weeks following delivery and usually without postinflammatory pigment change or scarring, even in darkskinned women [35, 41]. Sometimes it clears before birth [50]. The disease usually does not recur in following pregnancies or with menses or use of oral contraceptives $[4,34]$. If the disorder does recur (in approximately $10 \%$ of cases), it often begins early in pregnancy and is less extensive $[57 \bullet, 58]$.

\section{Prurigo of Pregnancy}

PP is a relatively common disease of pregnancy, with incidence of approximately 1 in 300 to 1 in 450 pregnancies $[28,59-61]$. It has been reported to occur in all trimesters, but it characteristically begins during midpregnancy (2530 weeks gestation) $[28,62]$. Prurigo can often persist for weeks or months after delivery [28]. The etiology of this condition is still unknown, although an association with atopy has been reported [17].

The disorder manifests with multiple discrete, small, erythematous, or skin-colored papules and nodules, which are exceptionally pruritic and often are excoriated [63]. The rash is usually located on the extensor surfaces of the legs and upper arms. The abdomen is sometimes affected. With time, the rash can become polymorphic with excoriated, crusted, and eczematous lesions but does not progress to vesicle formation [62]. Sometimes follicular lesions can develop [62].

There are no known adverse effects for the mother or the fetus [17]. Associations with intrahepatic cholestasis of pregnancy or an atopic tendency have been suggested [41]. It rarely recurs in subsequent pregnancies [63].

Laboratory tests are normal, although serum IgE levels might be elevated in some patients [62]. Immunofluorescence evaluation is negative [62]. Histopathology evaluation is not specific, and might reveal spongiosis, parakeratosis, and marked papillary dermal edema, findings which are identical to PUPPP.

Emollients, midpotency topical steroids, and oral antihistamines can be used for symptomatic treatment [17]. Systemic corticosteroids are only very seldom used [63].

\section{Pruritic Folliculitis of Pregnancy}

The incidence of PFP has been reported to be approximately 1:3,000 pregnancies [62]; however, this number is probably an underestimation and this condition is probably underreported, because it is commonly misdiagnosed as acne, bacterial folliculitis, or PUPPP [41].

The disorder presents at the second and third trimesters, usually between the fourth and ninth months of pregnancy $[41,62]$. It manifests as small $(3-5 \mathrm{~mm})$, multiple, disseminated, erythematous, excoriated, follicular papules and sterile pustules [41, 62]. The rash usually begins on the upper trunk and spreads to the extremities [62], and it strongly 
resembles steroid-induced acne. Despite its name, pruritus may not necessarily be present in PFP [41]. Spontaneous resolution occurs within weeks of delivery [41].

Histopathology is nonspecific and demonstrates characteristics of acute folliculitis consisting of intraluminal pustules with neutrophils, lymphocytes, macrophages, and eosinophils [62]. Mild superficial edema with a perivascular infiltrate of lymphohistiocytes and eosinophils is evident in the dermis [62]. Immunofluorescence studies are negative [62].

Etiology is still unclear [28], and there is no evidence to support immunologic or hormonal abnormalities [44•]. In the largest series of patients with PFP, there was a reported decrease in birth weight and a male/female ration of $2: 1$ [44•]. One case of PFP was associated with premature delivery [60], but maternal prognosis is good. Recurrence in subsequent pregnancies is rare [62].

Treatment includes topical low-potency corticosteroids, topical benzoyl peroxide $10 \%$, and ultraviolet B phototherapy $[28,62]$.

\section{Conclusions}

Skin symptoms are very common during pregnancy; most symptoms are mild and quickly resolve after delivery. Nevertheless, several specific dermatoses of pregnancy may have overwhelming effects on the patient's quality of life. Moreover, certain specific dermatoses of pregnancy (e.g., PG) may have adverse effects on the newborn. It is on this basis that good knowledge of these conditions is important for the treating clinician - gynecologist, dermatologist, or general practitioner. Accurate diagnosis and management is crucial for alleviating symptom of the pregnant woman, and in case of risk for the fetus, proper monitoring during pregnancy, and follow-up on the newborn's condition after delivery, are of utmost importance.

Disclosures The authors report no potential conflicts of interest relevant to this article.

\section{References}

Papers of particular interest, published recently, have been highlighted as:

- Of importance

•• Of major importance

1. Patsatsi A, Vavilis D, Tsikeloudi M, Kalabalikis D, Sotiriadis D. Refractory pemphigoid gestationis postpartum. Acta Obstet Gynecol Scand. 2012;91(5):636-7.

2. Shornick JK. Dermatoses of pregnancy. Semin Cutan Med Surg. 1998;17(3):172-81.
3. Shornick JK, Stastny P, Gilliam JN. High frequency of histocompatibility antigens HLA-DR3 and DR4 in herpes gestations. J Clin Invest. 1981;68(2):553-5.

4. Ambros-Rudolph CM. Dermatoses of pregnancy - clues to diagnosis, fetal risk and therapy. Ann Dermatol. 2011;23(3):265-75.

5. Lipozencic J, Ljubojevic S, Bukvic-Mokos Z. Pemphigoid gestationis. Clin Dermatol. 2012;30(1):51-5.

6. Kneisel A, Hertl M. Autoimmune bullous skin diseases. Part 1: Clinical manifestations. J Dtsch Dermatol Ges. 2011;9(10):84456. quiz 857.

7. Shornick JK, Bangert JL, Freeman RG, Gilliam JN. Herpes gestationis: clinical and histologic features of twenty-eight cases. J Am Acad Dermatol. 1983;8(2):214-24.

8. Semkova K, Black M. Pemphigoid gestationis: current insights into pathogenesis and treatment. Eur J Obstet Gynecol Reprod Biol. 2009; 145(2):138-44.

9. Rodrigues Cdos S, Filipe P, Solana Mdel M, Soares de Almeida L, Cirne de Castro J, Gomes MM. Persistent herpes gestationis treated with high-dose intravenous immunoglobulin. Acta Derm Venereol. 2007;87(2):184-6.

10. Horvath B, Niedermeier A, Podstawa E, Muller R, Hunzelmann N, Karpati S, et al. IgA autoantibodies in the pemphigoids and linear IgA bullous dermatosis. Exp Dermatol. 2010;19(7):648-53.

11. Noe MH, Messingham KA, Brandt DS, Andrews JI, Fairley JA. Pregnant women have increased incidence of $\operatorname{IgE}$ autoantibodies reactive with the skin and placental antigen BP180 (type XVII collagen). J Reprod Immunol. 2010;85(2):198-204.

12. •• Roger D, Vaillant L, Fignon A, Pierre F, Bacq Y, Brechot JF, et al. Specific pruritic diseases of pregnancy. A prospective study of 3192 pregnant women. Arch Dermatol. 1994;130(6):734-9. One of the most comprehensive study on pregnancy-specific dermatoses, which encompasses a wide array of diagnoses.

13. Panko J, Florell SR, Hadley J, Zone J, Leiferman K, Vanderhooft $\mathrm{S}$. Neonatal pemphigus in an infant born to a mother with serologic evidence of both pemphigus vulgaris and gestational pemphigoid. J Am Acad Dermatol. 2009;60(6):1057-62.

14. Jenkins RE, Hern S, Black MM. Clinical features and management of 87 patients with pemphigoid gestationis. Clin Exp Dermatol. 1999;24(4):255-9.

15. Nejim T, Jenkins CS, Uncles DR, Coburn P. Emergency caesarean delivery of a parturient with undiagnosed pemphigoid gestationis. Int J Obstet Anesth. 2012;21(3):288-90.

16. Di Zenzo G, Calabresi V, Grosso F, Caproni M, Ruffelli M, Zambruno G. The intracellular and extracellular domains of BP180 antigen comprise novel epitopes targeted by pemphigoid gestationis autoantibodies. J Invest Dermatol. 2007;127(4):86473.

17. Tunzi M, Gray GR. Common skin conditions during pregnancy. Am Fam Physician. 2007;75(2):211-8.

18. Chi CC, Wang SH, Charles-Holmes R, Ambros-Rudolph C, Powell J, Jenkins R, et al. Pemphigoid gestationis: early onset and blister formation are associated with adverse pregnancy outcomes. Br J Dermatol. 2009;160(6):1222-8.

19. Aoyama Y, Asai K, Hioki K, Funato M, Kondo N, Kitajima Y. Herpes gestationis in a mother and newborn: immunoclinical perspectives based on a weekly follow-up of the enzyme-linked immunosorbent assay index of a bullous pemphigoid antigen noncollagenous domain. Arch Dermatol. 2007;143(9):1168-72.

20. Bedocs PM, Kumar V, Mahon MJ. Pemphigoid gestationis: a rare case and review. Arch Gynecol Obstet. 2009;279(2):235-8.

21. Intong LR, Murrell DF. Pemphigoid gestationis: current management. Dermatol Clin. 2011;29(4):621-8.

22. Marker M, Derfler K, Monshi B, Rappersberger K. Successful immunoapheresis of bullous autoimmune diseases: pemphigus vulgaris and pemphigoid gestationis. J Dtsch Dermatol Ges. 2011;9(1):27-31. 
23. Cianchini G, Masini C, Lupi F, Corona R, De Pita O, Puddu P. Severe persistent pemphigoid gestationis: long-term remission with rituximab. Br J Dermatol. 2007;157(2):388-9.

24. Doiron P, Pratt M. Antepartum intravenous immunoglobulin therapy in refractory pemphigoid gestationis: case report and literature review. J Cutan Med Surg. 2010;14(4):189-92.

25. Bos JD. Reappraisal of dermatoses of pregnancy. Lancet. 1999;354 (9185): 1140.

26. Holmes RC, Black MM. The specific dermatoses of pregnancy. J Am Acad Dermatol. 1983;8(3):405-12.

27. Zoberman E, Farmer ER. Pruritic folliculitis of pregnancy. Arch Dermatol. 1981;117(1):20-2.

28. Kroumpouzos G, Cohen LM. Dermatoses of pregnancy. J Am Acad Dermatol. 2001;45(1):1-19. quiz 19-22.

29. Holmes RC, Black MM. The specific dermatoses of pregnancy: a reappraisal with special emphasis on a proposed simplified clinical classification. Clin Exp Dermatol. 1982;7(1):65-73.

30. Bourne G. Toxaemic rash of pregnancy. Proc R Soc Med. 1962;55:462-4.

31. Cooper AJ, Fryer JA. Prurigo of late pregnancy. Australas J Dermatol. 1980;21(2):79-84.

32. Holmes RC, Black MM, Dann J, James DC, Bhogal B. A comparative study of toxic erythema of pregnancy and herpes gestationis. Br J Dermatol. 1982;106(5):499-510.

33. Aronson IK, Bond S, Fiedler VC, Vomvouras S, Gruber D, Ruiz C. Pruritic urticarial papules and plaques of pregnancy: clinical and immunopathologic observations in 57 patients. J Am Acad Dermatol. 1998;39(6):933-9.

34. Lawley TJ, Hertz KC, Wade TR, Ackerman AB, Katz SI. Pruritic urticarial papules and plaques of pregnancy. JAMA. 1979;241 (16):1696-9.

35. Scheinfeld N. Pruritic urticarial papules and plaques of pregnancy wholly abated with one week twice daily application of fluticasone propionate lotion: a case report and review of the literature. Dermatol Online J. 2008;14(11):4.

36. High WA, Hoang MP, Miller MD. Pruritic urticarial papules and plaques of pregnancy with unusual and extensive palmoplantar involvement. Obstet Gynecol. 2005;105(5 Pt 2):1261-4.

37. - Rudolph CM, Al-Fares S, Vaughan-Jones SA, Mullegger RR, Kerl H, Black MM. Polymorphic eruption of pregnancy: clinicopathology and potential trigger factors in 181 patients. Br J Dermatol. 2006;154(1):54-60. A large study which highlights the major findings in PUPPP patients.

38. Brzoza Z, Kasperska-Zajac A, Oles E, Rogala B. Pruritic urticarial papules and plaques of pregnancy. J Midwifery Wom Health. 2007:52(1):44-8.

39. Sherley-Dale AC, Carr RA, Charles-Holmes R. Polymorphic eruption of pregnancy with bullous lesions: a previously unreported association. Br J Dermatol. 2010;162(1):220-2.

40. Kirkup ME, Dunnill MG. Polymorphic eruption of pregnancy developing in the puerperium. Clin Exp Dermatol. 2002;27 (8):657-60.

41. Kroumpouzos G, Cohen LM. Specific dermatoses of pregnancy: an evidence-based systematic review. Am J Obstet Gynecol. 2003;188(4):1083-92.

42. Aractingi S, Berkane N, Bertheau P, Le Goue C, Dausset J, Uzan $\mathrm{S}$, et al. Fetal DNA in skin of polymorphic eruptions of pregnancy. Lancet. 1998;352(9144):1898-901.

43. Cohen LM, Capeless EL, Krusinski PA, Maloney ME. Pruritic urticarial papules and plaques of pregnancy and its relationship to maternal-fetal weight gain and twin pregnancy. Arch Dermatol. 1989;125(11):1534-6.
44. - Vaughan Jones SA, Hern S, Nelson-Piercy C, Seed PT, Black MM. A prospective study of 200 women with dermatoses of pregnancy correlating clinical findings with hormonal and immunopathological profiles. Br J Dermatol. 1999;141(1):71-81. Although not very recent, this is an important study to understand the connection between dermatoses of pregnancy and hormonal and immunologic factors.

45. Alcalay J, Ingber A, Kafri B, Segal J, Kaufmann H, Hazaz B, et al. Hormonal evaluation and autoimmune background in pruritic urticarial papules and plaques of pregnancy. Am J Obstet Gynecol. 1988;158(2):417-20.

46. Ingber A, Alcalay J, Sandbank M. Multiple dermal fibroblasts in patients with pruritic urticarial papules and plaques of pregnancy. A clue to the etiology? Med Hypotheses. 1988;26(1): $11-2$.

47. Matz H, Orion E, Wolf R. Pruritic urticarial papules and plaques of pregnancy: polymorphic eruption of pregnancy (PUPPP). Clin Dermatol. 2006;24(2):105-8.

48. Sasseville D, Wilkinson RD, Schnader JY. Dermatoses of pregnancy. Int J Dermatol. 1981;20(4):223-41.

49. Campbell DM. Maternal adaptation in twin pregnancy. Semin Perinatol. 1986;10(1):14-8.

50. Callen JP, Hanno R. Pruritic urticarial papules and plaques of pregnancy (PUPPP). A clinicopathologic study. J Am Acad Dermatol. 1981;5(4):401-5.

51. Faber WR, van Joost T, Hausman R, Weenink GH. Late prurigo of pregnancy. Br J Dermatol. 1982;106(5):511-6.

52. Trattner A, Ingber A, Sandbank M. Antiepidermal cell surface antibodies in a patient with pruritic urticarial papules and plaques of pregnancy. J Am Acad Dermatol. 1991;24(2 Pt 1):306-8.

53. Petropoulou H, Georgala S, Katsambas AD. Polymorphic eruption of pregnancy. Int J Dermatol. 2006;45(6):642-8.

54. Woolf RT, Abdul-Wahab A. A pruritic rash in pregnancy. BMJ. 2011;343:d5325.

55. Yancey KB, Hall RP, Lawley TJ. Pruritic urticarial papules and plaques of pregnancy. Clinical experience in twenty-five patients. $\mathrm{J}$ Am Acad Dermatol. 1984;10(3):473-80.

56. Barrenetxea G, Melchor JC, Barbazan MJ, Aranguren G, Rodriguez-Escudero FJ. Pruritic urticarial papules and plaques of pregnancy. Int J Gynaecol Obstet. 1990;33(1):69-72.

57. • Ambros-Rudolph CM, Mullegger RR, Vaughan-Jones SA, Kerl $\mathrm{H}$, Black MM. The specific dermatoses of pregnancy revisited and reclassified: results of a retrospective two-center study on 505 pregnant patients. J Am Acad Dermatol. 2006;54(3):395-404. An important study on a large number of pregnant women, which helps to better elucidate the major findings in pregnancyassociated dermatoses.

58. Stollery N. Pregnancy related conditions. Practitioner. 2011;255 (1737):29-30.

59. Black MM. Prurigo of pregnancy, papular dermatitis of pregnancy, and pruritic folliculitis of pregnancy. Semin Dermatol. 1989;8 (1):23-5.

60. Reed J, George S. Pruritic folliculitis of pregnancy treated with narrowband (TL-01) ultraviolet B phototherapy. Br J Dermatol. 1999;141(1):177-9.

61. Vaughan Jones SA, Hern S, Black MM. Neutrophil folliculitis and serum androgen levels. Clin Exp Dermatol. 1999;24(5): 392-5.

62. Roth MM. Pregnancy dermatoses: diagnosis, management, and controversies. Am J Clin Dermatol. 2011;12(1):25-41.

63. Hayashi RH. Bullous dermatoses and prurigo of pregnancy. Clin Obstet Gynecol. 1990;33(4):746-53. 